\title{
Classes of functions associated with bounded Mocanu variation
}

\section{Jacek Dziok}

\author{
"Correspondence: jdziok@ur.edu.pl \\ Institute of Mathematics, University \\ of Rzeszów, Rzeszów, 35-310, Poland
}

\begin{abstract}
In the paper we introduce the class of linear combinations of functions which are subordinated to a convex function. Some relationships between this class and the class of real-valued functions with bounded variation on $[0,2 \pi]$ are obtained. Next, we define classes of functions associated with bounded Mocanu variation. By using the properties of multivalent prestarlike functions, we obtain various inclusion relationships between defined classes of functions. Some applications of the main results are also considered.
\end{abstract}

MSC: 30C45; 30C50; 30C55

Keywords: analytic functions; bounded variation; prestarlike function; Mocanu functions, linear operator; Hadamard product; subordination

\section{Introduction}

Let $\mathcal{A}$ denote the class of functions which are analytic in $\mathcal{U}:=\{z \in \mathbb{C}:|z|<1\}, \mathcal{A}_{0}:=\{f \in$ $\mathcal{A}: f(0)=1\}$, and let $\mathcal{A}_{p}(p \in \mathbb{N}:=\{1,2, \ldots\})$ denote the class of functions $f \in \mathcal{A}$ of the form

$$
f(z)=z^{p}+\sum_{n=p+1}^{\infty} a_{n} z^{n} \quad(z \in \mathcal{U}) .
$$

Mocanu [1] introduced the class $\mathcal{M}(\alpha)$ of functions $f \in \mathcal{A}_{1}$ such that $\frac{f(z) f^{\prime}(z)}{z} \neq 0(z \in \mathcal{U})$ and

$$
\operatorname{Re}\left\{(1-\alpha) \frac{z f^{\prime}(z)}{f(z)}+\alpha\left(1+\frac{z f^{\prime \prime}(z)}{f^{\prime}(z)}\right)\right\}>0 \quad(z \in \mathcal{U})
$$

In particular, $\mathcal{S}^{c}:=\mathcal{M}(1), \mathcal{S}^{*}:=\mathcal{M}(0)$ are the well-known classes of convex functions and starlike functions, respectively.

It is clear that $f \in \mathcal{S}^{c}$ if and only if $f$ is univalent in $\mathcal{U}$ and $f(\mathcal{U})$ is a convex domain. Also, by $\mathcal{S}_{0}^{c}$ we denote the class of functions $f \in \mathcal{A}_{0}$ which are univalent in $\mathcal{U}$ and $f(\mathcal{U})$ is a convex domain.

We say that a function $f \in \mathcal{A}_{1}$ is close-to-convex if there exists $g \in \mathcal{S}^{c}$ such that

$$
\operatorname{Re} \frac{f^{\prime}(z)}{g^{\prime}(z)}>0 \quad(z \in \mathcal{U}) .
$$

We denote by $\mathcal{C C}$ the class of all close-to-convex functions.

( 2013 Dziok; licensee Springer. This is an Open Access article distributed under the terms of the Creative Commons Attribution License (http://creativecommons.org/licenses/by/2.0), which permits unrestricted use, distribution, and reproduction in any medium, provided the original work is properly cited. 
We say that $f \in \mathcal{A}$ is subordinate to $F \in \mathcal{A}$, and we write $f(z) \prec F(z)$ (or simply $f \prec F$ ), if there exists a function

$$
\omega \in \Omega:=\{\omega \in \mathcal{A}:|\omega(z)| \leq|z|(z \in \mathcal{U})\},
$$

such that $f(z)=F(\omega(z))(z \in \mathcal{U})$. In particular, if $F$ is univalent in $\mathcal{U}$, we have the following equivalence

$$
f(z) \prec F(z) \quad \Longleftrightarrow \quad[f(0)=F(0) \wedge f(\mathcal{U}) \subset F(\mathcal{U})] .
$$

Let $h \in \mathcal{S}_{0}^{c}, \mu \geq 1$, and let us define

$$
\mathcal{K}_{\mu}(h):=\left\{\mu q_{1}+(1-\mu) q_{2}: q_{1}, q_{2} \prec h\right\} .
$$

We note that the class $\mathcal{P}:=\mathcal{K}_{1}\left(\frac{1+z}{1-z}\right)$ is the well-known class of Caratheodory functions.

Now we define generalizations of the classes $\mathcal{M}(\alpha)$ and $\mathcal{C C}$ associated with functions of bounded variation.

Let $p \in \mathbb{N}, \delta \in \mathbb{R}, \phi, \varphi, \xi \in \mathcal{A}_{p}, \Phi=(\phi, \varphi)$. We denote by $\mathcal{M}_{\mu}^{\delta}(\Phi, \xi, h)$ the class of functions $f \in \mathcal{A}_{p}$ such that

$$
(1-\delta) \frac{(\xi * \phi) * f}{(\xi * \varphi) * f}+\delta \frac{\phi * f}{\varphi * f} \in \mathcal{K}_{\mu}(h)
$$

where $*$ denotes the Hadamard product (or convolution). Moreover, let us define

$$
\begin{aligned}
& \mathcal{M}_{\mu}^{\delta}(\Phi, h):=\mathcal{M}_{\mu}^{\delta}\left(\Phi, \xi_{1}, h\right), \quad \mathcal{M}_{\mu}^{\delta}(\varphi, h):=\mathcal{M}_{\mu}^{\delta}\left(\left(\varphi_{2}, \varphi_{1}\right), h\right), \\
& \mathcal{W}_{\mu}(\Phi, h):=\mathcal{M}_{\mu}^{1}(\Phi, z, h), \quad \mathcal{W}_{\mu}(\varphi, h):=\mathcal{W}_{\mu}\left(\left(z \varphi^{\prime}(z) / p, \varphi\right), h\right), \\
& \mathcal{S}_{p}^{*}(\varphi, \alpha):=\mathcal{W}_{1}(\varphi,(1+(1-2 \alpha / p) z) /(1-z)),
\end{aligned}
$$

where

$$
\xi_{1}(z)=z^{p}+\sum_{n=p+1}^{\infty} \frac{p}{n} z^{n}, \quad \varphi_{1}(z)=\frac{z}{p} \varphi^{\prime}(z), \quad \varphi_{2}(z)=\frac{z}{p} \varphi_{1}^{\prime}(z) \quad(z \in \mathcal{U}) .
$$

Let $\boldsymbol{\mu}=\left(\mu_{1}, \mu_{2}\right), \mu_{1}, \mu_{2} \geq 1, \mathbf{h}=\left(h_{1}, h_{2}\right) \in \mathcal{S}_{0}^{c} \times \mathcal{S}_{0}^{c}$. We say that a function $f \in \mathcal{A}_{p}$ belongs to the class $\mathcal{C M}_{\mu}^{\delta}(\Phi, \xi, \mathbf{h})$ if there exists $g \in \mathcal{W}_{\mu_{2}}\left(\varphi, h_{2}\right)$ such that

$$
(1-\delta) \frac{(\xi * \phi) * f}{(\xi * \varphi) * g}+\delta \frac{\phi * f}{\varphi * g} \in \mathcal{K}_{\mu_{1}}\left(h_{1}\right)
$$

Moreover, let us define $\mathcal{C} \mathcal{W}_{\mu}(\Phi, h):=\mathcal{C M}_{\mu}^{1}\left(\Phi, z^{p}, h\right)$.

These general classes of functions reduce to well-known classes by judicious choices of the parameters; see, for example, [1-38]. In particular, the class $\mathcal{M}_{\mu}^{\delta}(\varphi, h)$ contains the functions $f \in \mathcal{A}_{p}$ such that

$$
L_{\delta}(f):=\frac{\delta}{p}\left(1+\frac{z(\varphi * f)^{\prime \prime}(z)}{(\varphi * f)^{\prime}(z)}\right)+(1-\delta) \frac{z(\varphi * f)^{\prime}(z)}{p(\varphi * f)(z)} \in \mathcal{K}_{\mu}(h) .
$$


It is related to the class of functions with bounded Mocanu variation defined by Coonce and Ziegler [6] and intensively investigated by Noor et al. [24-27]. The classes

$$
\mathcal{S}_{p}^{*}(\alpha):=\mathcal{S}_{p}^{*}\left(\frac{z^{p}}{1-z}, \frac{\alpha}{p}\right), \quad \mathcal{S}_{p}^{c}:=\mathcal{S}_{p}^{*}\left(\frac{z^{p}[p+(1-p) z]}{p(1-z)^{2}}, 0\right)
$$

are the classes of multivalent starlike functions of order $\alpha$ and multivalent convex functions, respectively. Choosing parameters

$$
\phi_{1}(z)=\frac{z}{1-z}, \quad \phi_{2}(z)=\frac{z}{(1-z)^{2}}, \quad h_{0}(z)=\frac{1+z}{1-z} \quad(z \in \mathcal{U})
$$

we obtain the well-known class $\mathcal{W}_{\mu}\left(\phi_{1}, h_{0}\right)$ of functions of bounded boundary rotation (see, for example, $[10,17,29,31,33])$. Moreover, it is clear that

$$
\mathcal{S}^{*}=\mathcal{S}_{1}^{*}(0), \quad \mathcal{S}^{c}=\mathcal{S}_{1}^{c}(0), \quad \mathcal{C} \mathcal{C}=\mathcal{C} \mathcal{W}_{(0,0)}\left(\left(\phi_{2}, \phi_{1}\right),\left(h_{0}, h_{0}\right)\right)
$$

The main object of this paper is to investigate convolution properties related to the prestarlike functions and various inclusion relationships between defined classes of functions. Some characterizations of the class $\mathcal{K}_{\mu}(h)$ are also given.

\section{Functions with bounded variation}

By $M_{k}(k \geq 2)$ we denote the class of real-valued functions $m$ of bounded variation on $[0,2 \pi]$ which satisfy the conditions

$$
\int_{0}^{2 \pi} \mathrm{d} m(t)=2, \quad \int_{0}^{2 \pi}|\mathrm{d} m(t)| \leq k,
$$

in terms of the Riemann-Stieltjes integral. It is clear that $M_{2}$ is the class of nondecreasing functions on $[0,2 \pi]$ satisfying (4) or, equivalently, $\int_{0}^{2 \pi} \mathrm{d} m(t)=2$.

The class $M_{k}$ is related to the class $\mathcal{K}_{\mu}(h)$ with $\mu=\frac{k}{4}+\frac{1}{2}$. Therefore, for the simplicity of notation, we define

$$
\mathcal{P}_{k}(h):=\mathcal{K}_{\mu}(h) \quad(\mu=k / 4+1 / 2, k \geq 2) .
$$

From the result of Hallenbeck and MacGregor ([18], p.50), we have the following lemma.

Lemma 1 Let $|c| \leq 1, c \neq-1, h(z)=\frac{1+c z}{1-z}(z \in \mathcal{U})$. Then $q \prec h$ if and only if there exists $m \in M_{2}$ such that

$$
q(z)=\frac{1}{2} \int_{0}^{2 \pi} h\left(z e^{-i t}\right) \mathrm{d} m(t) \quad(z \in \mathcal{U}) .
$$

Theorem 1 The class $\mathcal{K}_{\mu}(h)$ is convex.

Proof Let $q, r \in \mathcal{K}_{\mu}(h), \alpha \in[0,1]$. Then there exist $q_{j}, r_{j} \prec h(j=1,2)$ such that

$$
q=\mu q_{1}+(1-\mu) q_{2}, \quad r=\mu r_{1}+(1-\mu) r_{2} .
$$


Since

$$
\alpha q+(1-\alpha) r=\mu\left(\alpha q_{1}+(1-\alpha) r_{1}\right)+(1-\mu)\left(\alpha q_{2}+(1-\alpha) r_{2}\right)
$$

and $\alpha q_{j}+(1-\alpha) r_{j} \prec h(j=1,2)$, we conclude that $\alpha q+(1-\alpha) r \in \mathcal{K}_{\mu}(h)$. Hence, the class $\mathcal{K}_{\mu}(h)$ is convex.

Theorem 2 If $1 \leq \mu<\lambda$, then $\mathcal{K}_{\mu}(h) \subset \mathcal{K}_{\lambda}(h)$.

Proof Let $q \in \mathcal{K}_{\mu}(h)$. Then there exist $q_{1}, q_{2} \prec h$ such that $q=\mu q_{1}+(1-\mu) q_{2}$. Thus, we have

$$
q=\lambda q_{1}+(1-\lambda) \widetilde{q}_{2} \quad\left(\tilde{q}_{2}=\frac{\lambda-\mu}{\lambda-1} q_{1}+\frac{\mu-1}{\lambda-1} q_{2}\right) .
$$

Hence, we get $\widetilde{q}_{2} \prec h$ and, in consequence, $q \in \mathcal{K}_{\lambda}(h)$.

Theorem 3 Let $|c| \leq 1, c \neq-1, h(z)=\frac{1+c z}{1-z}(z \in \mathcal{U})$. Then $q \in \mathcal{P}_{k}(h)$ if and only if there exists $m \in M_{k}$ such that

$$
q(z)=\frac{1}{2} \int_{0}^{2 \pi} h\left(z e^{-i t}\right) \mathrm{d} m(t) \quad(z \in \mathcal{U})
$$

Proof Let $q \in \mathcal{P}_{k}(h)$. Then there exist $q_{1}, q_{2} \prec h$ such that $q=\left(\frac{k}{4}+\frac{1}{2}\right) q_{1}-\left(\frac{k}{4}-\frac{1}{2}\right) q_{2}$. Thus, by Lemma 1 there exist $m_{1}, m_{2} \in M_{2}$ such that

$$
q(z)=\frac{1}{2} \int_{0}^{2 \pi} h\left(z e^{-i t}\right) \mathrm{d} m(t) \quad\left(m=\left(\frac{k}{4}+\frac{1}{2}\right) m_{1}-\left(\frac{k}{4}-\frac{1}{2}\right) m_{2}\right)
$$

Since

$$
\int_{0}^{2 \pi} \mathrm{d} m(t)=\left(\frac{k}{4}+\frac{1}{2}\right) \int_{0}^{2 \pi} \mathrm{d} m_{1}(t)-\left(\frac{k}{4}-\frac{1}{2}\right) \int_{0}^{2 \pi} \mathrm{d} m_{2}(t)=2
$$

and

$$
\int_{0}^{2 \pi}|\mathrm{d} m(t)| \leq\left(\frac{k}{4}+\frac{1}{2}\right) \int_{0}^{2 \pi} \mathrm{d} m_{1}(t)+\left(\frac{k}{4}-\frac{1}{2}\right) \int_{0}^{2 \pi} \mathrm{d} m_{2}(t)=k,
$$

we have $m \in M_{k}$ and consequently (5). Conversely, let $q \in \mathcal{A}_{0}$ satisfy (5) for some $m \in M_{k}$. If $m \in M_{2}$, then by Lemma 1 and Theorem 2, we have $q \in \mathcal{P}_{2}(h) \subset \mathcal{P}_{k}(h)$. Let now $m \in$ $M_{k} \backslash M_{2}$. Since $m$ is the function with bounded variation, by the Jordan theorem there exist real-valued functions $\mu_{1}, \mu_{2}$ which are nondecreasing and nonconstant on $[0,2 \pi]$ such that

$$
m=\mu_{1}-\mu_{2}, \quad \int_{0}^{2 \pi}|\mathrm{d} m(t)|=\int_{0}^{2 \pi} \mathrm{d} \mu_{1}(t)+\int_{0}^{2 \pi} \mathrm{d} \mu_{2}(t) .
$$

Thus, putting

$$
\alpha_{j}=\frac{\mu_{j}(2 \pi)-\mu_{j}(0)}{2}, \quad m_{j}:=\frac{1}{\alpha_{j}} \mu_{j} \quad(j=1,2)
$$


we get $m_{1}, m_{2} \in M_{2}$ and

$$
m=\alpha_{1} m_{1}-\alpha_{2} m_{2}
$$

Combining (6) and (7), we obtain

$$
2 \alpha_{1}-2 \alpha_{2}=\int_{0}^{2 \pi} \mathrm{d} m(t)=2, \quad 2 \alpha_{1}+2 \alpha_{2}=\int_{0}^{2 \pi}|\mathrm{d} m(t)| \leq k
$$

and so

$$
\alpha_{1}=\left(\frac{\lambda}{4}+\frac{1}{2}\right), \quad \alpha_{2}=\left(\frac{\lambda}{4}-\frac{1}{2}\right) \quad\left(\lambda=\int_{0}^{2 \pi}|\mathrm{d} m(t)|, 2 \leq \lambda \leq k\right) .
$$

Therefore, by (5) and (7) we obtain

$$
q=\left(\frac{\lambda}{4}+\frac{1}{2}\right) q_{1}-\left(\frac{\lambda}{4}-\frac{1}{2}\right) q_{2}
$$

where

$$
q_{j}(z)=\frac{1}{2} \int_{0}^{2 \pi} h\left(z e^{-i t}\right) \mathrm{d} m_{j}(t) \quad(z \in \mathcal{U}, j=1,2) .
$$

Thus, by Lemma 1 and Theorem 2, we have $q \in \mathcal{P}_{\lambda}(h) \subset \mathcal{P}_{k}(h)$ and the proof is complete.

Let $\sigma_{h}$ denote the conformal mapping which maps $h(\mathcal{U})$ onto $\Pi:=\{w \in \mathbb{C}: \operatorname{Re} w>0\}$ with $\sigma_{h}(1)=1$ and let $D$ denote the set of analyticity of $\sigma_{h}$. Moreover, let us define

$$
\widetilde{\mathcal{A}}_{0}:=\left\{q \in \mathcal{A}_{0}: q(\mathcal{U}) \subset D\right\}, \quad \widetilde{\mathcal{P}}_{k}(h):=\left\{q \in \widetilde{\mathcal{A}}_{0}: \sigma_{h} \circ q \in \mathcal{P}_{k}\left(\frac{1+z}{1-z}\right)\right\}
$$

Lemma 2 Let $q \in \widetilde{\mathcal{A}}_{0}$. Then $q \in \mathcal{K}(h):=\mathcal{K}_{2}(h)$ if and only if

$$
\int_{0}^{2 \pi}\left|\operatorname{Re}\left(\sigma_{h} \circ q\right)\left(r e^{i t}\right)\right| \mathrm{d} t=2 \pi \quad(0<r<1) .
$$

Proof Let $q \in \widetilde{\mathcal{A}}_{0}$. Then, by the properties of subordination, we have

$$
\begin{aligned}
q \in \mathcal{K}(h) & \Longleftrightarrow q \prec h \quad \Longleftrightarrow \quad \sigma_{h} \circ q \prec \sigma_{h} \circ h \\
& \Longleftrightarrow \operatorname{Re}\left(\sigma_{h} \circ q\right)(z)>0 \quad(z \in \mathcal{U}) .
\end{aligned}
$$

Moreover,

$$
\int_{0}^{2 \pi} \operatorname{Re}\left(\sigma_{h} \circ q\right)\left(r e^{i t}\right) \mathrm{d} t=\operatorname{Re} \int_{|z|=r} \frac{\left(\sigma_{h} \circ q\right)(z)}{z} i \mathrm{~d} z=2 \pi\left(\sigma_{h} \circ q\right)(0)=2 \pi \quad(0<r<1) .
$$

Thus, condition (8) is equivalent to $\operatorname{Re}\left(\sigma_{h} \circ q\right)(z)>0(z \in \mathcal{U})$ and by (9) we have, equivalently, $q \in \mathcal{K}(h)$. 
Let $\mathcal{H}(D), \mathcal{S H}(D)$ denote the classes of harmonic and subharmonic functions in the domain $D \subset \mathbb{C}$, respectively.

Theorem 4 Let $q \in \widetilde{\mathcal{A}}_{0}$. Then $q \in \widetilde{\mathcal{P}}_{k}(h)$ if and only if

$$
\int_{0}^{2 \pi}\left|\operatorname{Re}\left(\sigma_{h} \circ q\right)\left(r e^{i t}\right)\right| \mathrm{d} t \leq k \pi \quad(0<r<1)
$$

Proof Let $q \in \widetilde{\mathcal{P}}_{k}(h)$. Then there exist $q_{1}, q_{2} \prec \frac{1+z}{1-z}$ such that $\sigma_{h} \circ q=\left(\frac{k}{4}+\frac{1}{2}\right) q_{1}-\left(\frac{k}{4}-\frac{1}{2}\right) q_{2}$. Hence, by Lemma 2 we have

$$
\begin{aligned}
\int_{0}^{2 \pi}\left|\operatorname{Re}\left(\sigma_{h} \circ q\right)\left(r e^{i t}\right)\right| \mathrm{d} t \leq & \left(\frac{k}{4}+\frac{1}{2}\right) \int_{0}^{2 \pi}\left|\operatorname{Re} q_{1}\left(r e^{i t}\right)\right| \mathrm{d} t \\
& +\left(\frac{k}{4}-\frac{1}{2}\right) \int_{0}^{2 \pi}\left|\operatorname{Re} q_{2}\left(r e^{i t}\right)\right| \mathrm{d} t=k \pi \quad(0<r<1) .
\end{aligned}
$$

To obtain the converse, suppose that $q \in \widetilde{\mathcal{A}}_{0}$ satisfies (10). By Lemma 2 we can assume $k>2$. If we put

$$
\begin{aligned}
& F:=\operatorname{Re}\left(\sigma_{h} \circ q\right), \quad F^{+}(z):=\max \{F(z), 0\} \geq 0, \\
& F^{-}(z):=\max \{-F(z), 0\} \geq 0 \quad(z \in \mathcal{U}), \\
& V_{r}^{\tau}(z):=\frac{1}{2 \pi} \int_{0}^{2 \pi} \frac{r^{2}-|z|^{2}}{\left|r e^{i t}-z\right|^{2}} F^{\tau}\left(r e^{i t}\right) \mathrm{d} t \geq 0 \quad(|z| \leq r<1, \tau \in\{+,-\}),
\end{aligned}
$$

then the functions $F^{\tau}, V_{r}^{\tau}(\tau \in\{+,-\})$ are nonconstant and

$$
\begin{aligned}
& F \in \mathcal{H}(\mathcal{U}), \quad V_{r}^{+}, V_{r}^{-} \in \mathcal{H}\left(\mathcal{U}_{r}\right), \quad F^{+}, F^{-} \in \mathcal{S H}(\mathcal{U}), \\
& F=F^{+}-F^{-}, \quad|F|=F^{+}+F^{-}, \quad V_{r}^{\tau}(z)=F^{\tau}(z) \quad(|z|=r, \tau \in\{+,-\}) .
\end{aligned}
$$

Thus, we have

$$
\begin{aligned}
\max \left\{F^{\tau}(z), V_{r}^{\tau}(z)\right\} & =V_{r}^{\tau}(z) \quad(|z| \leq r, r \in(0,1), \tau \in\{+,-\}), \\
\max \left\{V_{r}^{\tau}(z):|z| \leq r\right\} & =\max \left\{F^{\tau}(z):|z|=r\right\} \\
& \leq \max \left\{F^{\tau}(z):|z| \leq R\right\} \quad(r \leq R<1, \tau \in\{+,-\}) .
\end{aligned}
$$

Therefore, the functions

$$
G_{r}^{\tau}(z):=\left\{\begin{array}{ll}
V_{r}^{\tau}(z), & |z|<r, \\
F^{\tau}(z), & r \leq|z|<1
\end{array} \quad(r \in(0,1), \tau \in\{+,-\}),\right.
$$

are continuous subharmonic functions in $\mathcal{U}$ and the families $\left\{G_{r}^{+}: r \in(0,1)\right\},\left\{G_{r}^{-}: r \in\right.$ $(0,1)\}$ are locally uniformly bounded. Hence, if we define

$$
G^{\tau}(z):=\sup \left\{G_{r}^{\tau}(z): r \in(0,1)\right\}=\lim _{n \rightarrow \infty} G_{1-\frac{1}{n}}^{\tau}(z) \quad(z \in \mathcal{U}, \tau \in\{+,-\})
$$


then

$$
G^{\tau} \in \mathcal{S H}(\mathcal{U}), \quad G_{r}^{\tau}, G^{\tau} \in \mathcal{H}\left(\mathcal{U}_{r}\right) \quad(r \in(0,1), \tau \in\{+,-\})
$$

and, in consequence, $G^{+}, G^{-} \in \mathcal{H}(\mathcal{U}), G^{+}, G^{-}>0$. Moreover, by (11) we get

$$
\begin{aligned}
& F(z)=G_{r}^{+}(z)-G_{r}^{-}(z) \quad(|z| \leq r, r \in(0,1)), \\
& |F(z)|=G_{r}^{+}(z)+G_{r}^{-}(z) \quad(|z|=r, r \in(0,1)) .
\end{aligned}
$$

Therefore, we have

$$
F(z)=\alpha_{1} G_{1}(z)-\alpha_{2} G_{2}(z) \quad(z \in \mathcal{U}),
$$

where

$$
G_{1}:=\frac{1}{\alpha_{1}} G^{+}, \quad G_{2}:=\frac{1}{\alpha_{2}} G^{-} \quad\left(\alpha_{1}=G^{+}(0), \alpha_{2}=G^{-}(0)\right)
$$

are positive harmonic functions in $\mathcal{U}$. Moreover, by (12) we obtain

$$
\lim _{r \rightarrow 1^{-}} \int_{0}^{2 \pi}\left|F\left(r e^{i t}\right)\right| \mathrm{d} t=\alpha_{1} \lim _{r \rightarrow 1^{-}} \int_{0}^{2 \pi} G_{1}\left(r e^{i t}\right) \mathrm{d} t+\alpha_{2} \lim _{r \rightarrow 1^{-}} \int_{0}^{2 \pi} G_{2}\left(r e^{i t}\right) \mathrm{d} t .
$$

Now, we consider functions $q_{1}, q_{2} \in \mathcal{A}_{0}$ such that

$$
\operatorname{Re} q_{j}(z)=G_{j}(z)>0 \quad(z \in \mathcal{U}, j=1,2) .
$$

Then $q_{1}, q_{2} \prec \frac{1+z}{1-z}$ and by (13) we obtain

$$
\sigma_{h} \circ q=\alpha_{1} q_{1}-\alpha_{2} q_{2}
$$

Hence, we get $\alpha_{1}-\alpha_{2}=1$. Moreover, by (14) and Lemma 2, we have $2 \alpha_{1}+2 \alpha_{2}=\lambda$, where $\lambda=: \frac{1}{\pi} \lim _{r \rightarrow 1^{-}} \int_{0}^{2 \pi}\left|F\left(r e^{i t}\right)\right| \mathrm{d} t$ and $2 \leq \lambda \leq k$, by (10). Thus, we get

$$
\alpha_{1}=\frac{\lambda}{4}+\frac{1}{2}, \quad \alpha_{2}=\frac{\lambda}{4}-\frac{1}{2}, \quad 2 \leq \lambda \leq k .
$$

Therefore, by (15) and Theorem 2, we have $q \in \widetilde{\mathcal{P}}_{\lambda}(h) \subset \widetilde{\mathcal{P}}_{k}(h)$, which proves the theorem.

If $h(z)=\frac{1+(1-2 a) z}{1-z}, \sigma_{h}(z)=\frac{z-a}{1-a}(z \in \mathcal{U}, a \neq 0)$, then $\widetilde{\mathcal{P}}_{k}(h)=\mathcal{P}_{k}(h)$. Thus, by Theorem 4 we get the following result.

Corollary $1[10]$ Let $q \in \mathcal{A}_{0}, h(z)=\frac{1+(1-2 a) z}{1-z}, a \neq 1$. Then $q \in \mathcal{P}_{k}(h)$ if and only if (10) holds.

Remark 1 Theorem 3 and Theorem 4 give relationships between the class $\mathcal{P}_{k}(h)$ and classes investigated by Paatero [29], Pinchuk [33], Padmanabhan and Parvatham [31] and Moulis [21] (for the precise relationships, see Dziok [10]). 
The first-order differential subordination

$$
q(z)+\frac{z q^{\prime}(z)}{\beta q(z)+\gamma} \prec h(z)
$$

is called the Briot-Bouquet differential subordination. This particular differential subordination has a surprising number of important applications in the theory of analytic functions (for details, see [20]). In particular, Eenigenburg, Miller, Mocanu and Reade [14] proved the following result.

Lemma 3 Let $h \in \mathcal{S}_{0}^{c}, \beta, \gamma \in \mathbb{C}$, and $\operatorname{Re}(\beta h(z)+\gamma)>0(z \in \mathcal{U})$. If $q \in \mathcal{A}_{0}$ satisfies the BriotBouquet differential subordination (16), then $q \prec h$.

For $\beta=0$ we can extend this result.

Theorem 5 Let $q \in \mathcal{A}_{0}, \operatorname{Re} \gamma>0$. If

$$
q(z)+\gamma z q^{\prime}(z) \in K_{\mu}(h)
$$

then $q \in K_{\mu}(h)$.

Proof From (17) there exist $q_{1}, q_{2} \prec h$ such that

$$
q(z)+\gamma z q^{\prime}(z)=\mu q_{1}(z)+(1-\mu) q_{2}(z) \quad(z \in \mathcal{U}) .
$$

Let $\widetilde{q}_{1}, \widetilde{q}_{2}$ be the solutions of the Cauchy problems

$$
\begin{array}{ll}
\widetilde{q}(z)+\gamma z \widetilde{q}^{\prime}(z)=q_{1}(z), & \widetilde{q}(0)=1, \\
\widetilde{q}(z)+\gamma z \widetilde{q}^{\prime}(z)=q_{2}(z), & \widetilde{q}(0)=1,
\end{array}
$$

respectively. Then, by (18) we have $q=\mu \widetilde{q}_{1}+(1-\mu) \widetilde{q}_{2}$. Moreover, by Lemma 3 we get $\widetilde{q}_{1}, \widetilde{q}_{2} \prec h$. Therefore, $q \in K_{\mu}(h)$ and the proof is completed.

A more general problem can be formulated as the following problem.

Problem 1 Let $\operatorname{Re}(\beta h(z)+\gamma)>0(z \in \mathcal{U})$. To verify the following result: if $q \in \mathcal{A}_{0}$ satisfies

$$
q(z)+\frac{z q^{\prime}(z)}{\beta q(z)+\gamma} \in \mathcal{K}_{\mu}(h)
$$

then $q \in \mathcal{K}_{\mu}(h)$.

Remark 2 The result from the problem was used in some papers (see, for example, [3, 23, 26 ] and [27]). It is clear, by Theorem 5 , that the result is true for $\beta=0$, but for $\beta \neq 0$ it is the open problem. 


\section{Properties of multivalent prestarlike functions}

Let $\alpha<p$. We say that a function $f \in \mathcal{A}_{p}$ belongs to the class $\mathcal{R}_{p}(\alpha)$ of multivalent prestarlike functions of order $\alpha$ if

$$
f(z) * \frac{z^{p}}{(1-z)^{2(p-\alpha)}} \in \mathcal{S}_{p}^{*}(\alpha) .
$$

It is easy to verify that

$$
\mathcal{R}_{p}(\alpha)=\mathcal{W}_{1}\left(\frac{z^{p}}{(1-z)^{2(p-\alpha)}}, \frac{1+(1-2 \alpha / p) z}{1-z}\right) .
$$

The class $\mathcal{R}(\alpha):=\mathcal{R}_{1}(\alpha)$ is the well-known class of prestarlike functions of order $\alpha$ introduced by Ruscheweyh [35].

We denote the dual set of $\mathcal{V} \subset \mathcal{A}_{0}$ by $\mathcal{V}^{*}:=\left\{q \in \mathcal{A}_{0}:(r * q)(z) \neq 0(r \in \mathcal{V}, z \in \mathcal{U})\right\}$. Moreover, let us define

$$
\begin{aligned}
& \mathcal{T}(\beta):=\left\{\frac{(1+x z)}{(1+y z)^{\beta}}:|x|=|y|=1\right\} \quad(\beta \geq 0), \\
& \mathcal{H}:=[\mathcal{T}(1)]^{*}, \quad \mathcal{H}^{k}:=\left\{q^{k}: q \in \mathcal{H}\right\} \quad(k \geq 0), \\
& \mathcal{F}(0, \lambda):=\left\{q \in \mathcal{A}_{0}: \operatorname{Re}\left(\frac{z q^{\prime}(z)}{q(z)}\right)>-\frac{\lambda}{2}(z \in \mathcal{U})\right\}, \\
& \mathcal{F}(k, \beta):=\left\{r q: r \in \mathcal{H}^{k} \text { and } q \in \mathcal{F}(0, \beta-k)\right\} \quad(0 \leq k \leq \beta) .
\end{aligned}
$$

It is clear that

$$
\mathcal{V}_{1} \subset \mathcal{V}_{2} \quad \Longrightarrow \quad \mathcal{V}_{2}^{*} \subset \mathcal{V}_{1}^{*}
$$

and

$$
0 \leq k \leq \beta_{1} \leq \beta_{2} \quad \Longrightarrow \quad \mathcal{F}\left(k, \beta_{1}\right) \subset \mathcal{F}\left(k, \beta_{2}\right) \text {. }
$$

In proving our main results, we need the following lemmas.

Lemma 4 [19] Let $w$ be a nonconstant function meromorphic in $\mathcal{U}$ with $w(0)=0$. If

$$
\left|w\left(z_{0}\right)\right|=\max \left\{|w(z)| ;|z| \leq\left|z_{0}\right|\right\}
$$

for some $z_{0} \in \mathcal{U}$, then there exists a real number $k(k \geq 1)$ such that $z_{0} w^{\prime}\left(z_{0}\right)=k w\left(z_{0}\right)$.

Lemma 5 ([34], p.29) Let $\beta \geq 0$ and $f \in \mathcal{A}_{0}$. Then $f \in[\mathcal{T}(\beta)]^{*}$ if and only if

$$
\left\{\begin{array}{l}
\operatorname{Re} \frac{z f_{\beta}^{\prime}(z)}{f_{\beta}(z)}>\frac{1-\beta}{2} \quad \text { for } \beta \neq 1, \\
\operatorname{Re} f(z)>\frac{1}{2} \quad \text { for } \beta=1
\end{array} \quad(z \in \mathcal{U}),\right.
$$

where

$$
f_{\beta}(z)=f(z) *(1-z)^{1-\beta} \quad(z \in \mathcal{U}) .
$$


Lemma 6 ([34], p.39) Let $\beta \geq 1$. If $f, g \in[\mathcal{T}(\beta)]^{*}$, then $f * g \in[\mathcal{T}(\beta)]^{*}$.

Lemma 7 ([34], p.20 and p.33) If $\beta \geq 1$, then $[\mathcal{F}(1, \beta)]^{*}=[\mathcal{T}(\beta)]^{*}$.

Lemma 8 ([34], p.37) Let $\beta \geq 1, f \in[\mathcal{T}(\beta)]^{*}, g \in \mathcal{F}(0, \beta-1), q \in \mathcal{A}$. Then

$$
\frac{f *(q g)}{f * g}(\mathcal{U}) \subseteq \overline{c o}\{q(\mathcal{U})\}
$$

where $\overline{c o}\{q(\mathcal{U})\}$ denotes the closed convex hull of $q(\mathcal{U})$.

From Lemma 5 and definitions of the classes $\mathcal{R}_{p}(\alpha), \mathcal{S}_{p}^{*}(\alpha)$ and $\mathcal{F}(\alpha, \beta)$ we obtain the following two results.

Lemma 9 A function $f$ belongs to the class $\mathcal{R}_{p}(\alpha)$ if and only if

$$
\frac{f(z)}{z^{p}} \in[\mathcal{T}(2 p+1-2 \alpha)]^{*}
$$

Lemma 10 A function $f$ belongs to the class $\mathcal{S}_{p}^{*}(\alpha)$ if and only if

$$
\frac{f(z)}{z^{p}} \in \mathcal{F}(0,2 p-2 \alpha)
$$

Using Lemmas 6 and 9, we have the following theorem.

Theorem 6 Iff, $g \in \mathcal{R}_{p}(\alpha)$, then $f * g \in \mathcal{R}_{p}(\alpha)$.

Theorem 7 If $\alpha_{1} \leq \alpha_{2}<p$, then

$$
\mathcal{R}_{p}\left(\alpha_{1}\right) \subset \mathcal{R}_{p}\left(\alpha_{2}\right)
$$

Proof By condition (20) we have

$$
\mathcal{F}\left(1,2 p+1-2 \alpha_{2}\right) \subset \mathcal{F}\left(1,2 p+1-2 \alpha_{1}\right) .
$$

Thus, by (19) and Lemma 7, we obtain

$$
\begin{aligned}
{\left[\mathcal{T}\left(2 p+1-2 \alpha_{1}\right)\right]^{*} } & =\left[\mathcal{F}\left(1,2 p+1-2 \alpha_{1}\right)\right]^{*} \subset\left[\mathcal{F}\left(1,2 p+1-2 \alpha_{2}\right)\right]^{*} \\
& =\left[\mathcal{T}\left(2 p+1-2 \alpha_{2}\right)\right]^{*}
\end{aligned}
$$

and by Lemma 9 we get the inclusion relation (22).

Making use of Lemmas 8-10, we get the following theorem.

Theorem 8 Let $f \in \mathcal{R}_{p}(\alpha), g \in \mathcal{S}_{p}^{*}(\alpha)$. Then (21) holds for $q \in \mathcal{A}$. 
For complex parameters $a, b, c(c \neq 0,-1,-2, \ldots)$, the hypergeometric function ${ }_{2} F_{1}(a, b ; c ; z)$ is defined by

$$
{ }_{2} F_{1}(a, b ; c ; z):=\sum_{n=0}^{\infty} \frac{(a)_{n}(b)_{n}}{(c)_{n} n !} z^{n} \quad(z \in \mathcal{U}),
$$

where

$$
(\lambda)_{n}:= \begin{cases}1 & (n=0), \\ \lambda(\lambda+1) \cdots(\lambda+n-1) & (n \in \mathbb{N})\end{cases}
$$

is the Pochhammer symbol. Next, we define

$$
\varphi_{p}(a, b ; c)(z):=z_{2}^{p} F_{1}(a, b ; c ; z) \quad(z \in \mathcal{U}) .
$$

In particular, the function

$$
l_{p}(a, c)(z):=\varphi_{p}(a, 1 ; c)(z) \quad(z \in \mathcal{U})
$$

will be called the multivalent incomplete hypergeometric function.

\section{Theorem 9 If either}

$$
\operatorname{Re} a \leq \operatorname{Re} c, \quad \operatorname{Im} a=\operatorname{Im} c \text { and } \quad(2 p+1-a-\bar{c}) / 2 \leq \alpha<p
$$

or

$$
0<a \leq c \text { and }\left(p-\frac{c}{2}\right) \leq \alpha<p,
$$

then

$$
l_{p}(a, c) \in \mathcal{R}_{p}(\alpha) .
$$

Proof Let (24) hold true. By Theorem 7 it is sufficient to prove (26) for $\alpha=\frac{1}{2}(2 p+1-a-\bar{c})$. It is easy to show that

$$
\varphi_{p}(a, b ; c)=l_{p}(a, c) * \varphi_{p}(1, b ; 1)
$$

and

$$
\varphi_{p}(1 ; 2 p-2 \alpha ; 1)(z)=\frac{z^{p}}{(1-z)^{2(p-\alpha)}} \quad(z \in \mathcal{U}) .
$$

Thus, condition (26) is equivalent to $\varphi_{p}(a, b ; c) \in \mathcal{S}_{p}^{*}(\alpha)$, where $b=a+\bar{c}-1>0$. Using the notation $F(z):={ }_{2} F_{1}(a, b ; c ; z)(z \in \mathcal{U})$, we have to show that

$$
\operatorname{Re}\left(\frac{z F^{\prime}(z)}{F(z)}\right)>-\frac{b}{2} \quad(z \in \mathcal{U})
$$


or that the meromorphic function $\omega, \omega(0)=0$, defined by

$$
\frac{z F^{\prime}(z)}{F(z)}=b \frac{\omega(z)}{1-\omega(z)} \quad(z \in \mathcal{U})
$$

is bounded by 1 in $\mathcal{U}$. If this is false, we find $z_{0} \in \mathcal{U}$ such that $\left|w\left(z_{0}\right)\right|=1,|w(z)|<1\left(|z|<\left|z_{0}\right|\right)$. According to Lemma 4 , there exists $k \geq 1$ such that

$$
z_{0} w^{\prime}\left(z_{0}\right)=k w\left(z_{0}\right), \quad w\left(z_{0}\right)=e^{i \theta}
$$

Taking the logarithmic derivative of (27), we get

$$
\frac{z F^{\prime \prime}(z)}{F^{\prime}(z)}=\frac{(b+1) \omega(z)-1}{1-\omega(z)}+\frac{z \omega^{\prime}(z)}{[1-\omega(z)] \omega(z)} \quad(z \in \mathcal{U})
$$

The hypergeometric function $F$ satisfies the Gaussian hypergeometric differential equation

$$
z(1-z) F^{\prime \prime}+[c-(a+b+1) z] F^{\prime}-a b F=0 .
$$

Therefore, by (27) and (29), we obtain

$$
z A(z)=B(z) \quad(z \in \mathcal{U})
$$

where

$$
A(z)=a+\left(b-a+\frac{\left.z \omega{ }^{\prime} z\right)}{\omega(z)}\right) \omega(z), \quad B(z)=\omega(z)\left[\frac{\left.z \omega{ }^{\prime} z\right)}{\omega(z)}+c-1+(b+1-c) \omega(z)\right] .
$$

Thus, by (28) we have

$$
A\left(z_{0}\right)=a+(k-1+\bar{c}) e^{i \theta}, \quad B\left(z_{0}\right)=k-1+c+\bar{a} e^{i \theta} .
$$

Since $A\left(z_{0}\right)=e^{i \theta} \overline{B\left(z_{0}\right)}$, we have $\left|A\left(z_{0}\right)\right|=\left|B\left(z_{0}\right)\right|$. Furthermore, $A\left(z_{0}\right) \neq 0$ under restrictions (24). Thus (30) gives $\left|z_{0}\right|=1$, a contradiction. Now let (25) hold true. It is clear that the function

$$
l_{p}(a, 1)(z)=\frac{z^{p}}{(1-z)^{a}} \quad(z \in \mathcal{U})
$$

belongs to the class $\mathcal{S}_{p}^{*}\left(p-\frac{a}{2}\right) \subset \mathcal{S}_{p}^{*}\left(p-\frac{c}{2}\right)$ and $l_{p}(a, c) * l_{p}(c, 1)=l_{p}(a, 1)$. Thus, by the definition of the class $\mathcal{R}_{p}(\alpha)$ and Theorem 7, we have $l_{p}(a, c) \in \mathcal{R}_{p}\left(p-\frac{c}{2}\right) \subset \mathcal{R}_{p}(\alpha)$.

Remark 3 The results related to multivalent prestarlike functions were obtained in the submitted paper [7]. Since the paper has not been accepted for publication so far, these results are presented with the proofs. 


\section{The main inclusion relationships}

From now on we make the assumptions:

$$
\operatorname{Re} h(z)>\alpha, \quad \operatorname{Re} h_{j}(z)>\alpha \quad(z \in \mathcal{U}, j=1,2) .
$$

Moreover, let $\psi *(\phi, \varphi):=(\psi * \phi, \psi * \varphi)$. Then we have

$$
\mathcal{W}_{\mu}(h):=\mathcal{W}_{\mu}\left(z^{p} /(1-z), h\right) \subset \mathcal{S}_{p}^{*}(\alpha) \quad \text { and } \quad \mathcal{W}_{\mu}(\varphi, h) \subset \mathcal{S}_{p}^{*}(\varphi, \alpha)
$$

Lemma 11 If $0 \leq \lambda<\delta$, then

$$
\mathcal{M}_{1}^{\delta}(\varphi, h) \subset \mathcal{M}_{1}^{\lambda}(\varphi, h) \subset \mathcal{W}_{1}(\varphi, h)
$$

Proof Let $f \in \mathcal{M}_{1}^{\delta}(\varphi, h)$ and let

$$
q(z):=\frac{z(\varphi * f)^{\prime}(z)}{p(\varphi * f)(z)} \quad(z \in \mathcal{U})
$$

Then we obtain

$$
q(z)+\frac{\delta}{p} \frac{z q^{\prime}(z)}{q(z)}=L_{\delta}(f)(z) \quad(z \in \mathcal{U})
$$

where $L_{\delta}$ is defined by (3). Since $L_{\delta}(f) \prec h$, by Lemma 3 we have $q \prec h$. Moreover,

$$
L_{\lambda}(f)=\frac{\lambda}{\delta} L_{\delta}(f)+\frac{\delta-\lambda}{\delta} q .
$$

Thus, by Theorem 1 we get $L_{\lambda}(f) \prec h$ or, equivalently, $f \in \mathcal{M}_{1}^{\lambda}(\varphi, h)$.

Theorem 10 If $\psi \in \mathcal{R}_{p}(\alpha)$, then

$$
\begin{aligned}
& \mathcal{W}_{\mu}(\Phi, h) \cap \mathcal{W}_{\mu}(\varphi, h) \subset \mathcal{W}_{\mu}(\psi * \Phi, h), \\
& \mathcal{W}_{\mu}(\varphi, h) \subset \mathcal{W}_{\mu}(\psi * \varphi, h) .
\end{aligned}
$$

Proof Let $f \in \mathcal{W}_{\mu}(\Phi, h) \cap \mathcal{W}_{\mu}(\varphi, h)$. Then there exist $\omega_{1}, \omega_{2} \in \Omega$ such that

$$
\frac{\phi * f}{\varphi * f}=\mu\left(h \circ \omega_{1}\right)+(1-\mu)\left(h \circ \omega_{2}\right)
$$

and $F=\varphi * f \in \mathcal{W}_{\mu}(h) \subset \mathcal{S}_{p}^{*}(\alpha)$. Thus, applying the properties of convolution, we get

$$
\frac{(\psi * \phi) * f}{(\psi * \varphi) * f}=\mu \frac{\psi *\left[\left(h \circ \omega_{1}\right) F\right]}{\psi * F}+(1-\mu) \frac{\psi *\left[\left(h \circ \omega_{2}\right) F\right]}{\psi * F} .
$$

By Theorem 8 we conclude that

$$
q_{j}(z):=\frac{\psi *\left[\left(h \circ \omega_{j}\right) F\right]}{\psi * F}(z) \in \overline{c o}\{h(\omega(\mathcal{U}))\} \subset \overline{h(\mathcal{U})} \quad(z \in \mathcal{U}, j=1,2) .
$$


Therefore, $q_{j} \prec h$ and by (35) we have $f \in \mathcal{W}_{\mu}(\psi * \Phi, h)$, which proves the inclusion (33). To prove (34), we assume that $f \in \mathcal{W}_{\mu}(\varphi, h)$. Then $f \in \mathcal{W}_{\mu}((\phi, \varphi), h)$, where $\phi(z)=\frac{z}{p} \varphi^{\prime}(z)(z \in$ $\mathcal{U})$. Thus, by (33) we obtain that $f \in \mathcal{W}_{\mu}((\psi * \phi, \psi * \varphi), h)$. Since $(\psi * \phi)(z)=\frac{z}{p}(\psi * \varphi)^{\prime}(z)$ $(z \in \mathcal{U})$, we have $f \in \mathcal{W}_{\mu}(\psi * \varphi, h)$, which proves (34) and completes the proof.

Theorem 11 Let $\psi, \xi \in \mathcal{R}_{p}(\alpha), 0 \leq \delta \leq 1$. Then

$$
\begin{aligned}
& \mathcal{M}_{\mu}^{\delta}(\Phi, \xi, h) \cap \mathcal{W}_{\mu}(\Phi, h) \subset \mathcal{M}_{\mu}^{\delta}(\psi * \Phi, \xi, h), \\
& \mathcal{M}_{1}^{\delta}(\varphi, h) \subset \mathcal{M}_{1}^{\delta}(\psi * \varphi, h) .
\end{aligned}
$$

Proof Let $\mathcal{M}_{\mu}^{\delta}(\Phi, \xi, h) \cap \mathcal{W}_{\mu}(\Phi, h)$. Then, applying Theorem 6 and Theorem 10 , we obtain $f \in \mathcal{W}_{\mu}(\psi * \Phi, h)$ and $f \in \mathcal{W}_{\mu}(\xi * \psi * \Phi, h)$ or, equivalently,

$$
q_{1}:=\frac{\phi * \psi * f}{\varphi * \psi * f} \in \mathcal{K}_{\mu}(h), \quad q_{2}:=\frac{\xi * \phi * \psi * f}{\xi * \varphi * \psi * f} \in \mathcal{K}_{\mu}(h)
$$

Since the class $\mathcal{K}_{\mu}(h)$ is convex by Theorem 1 , we conclude that

$$
(1-\delta) \frac{\xi *(\psi * \phi) * f}{\xi *(\psi * \varphi) * f}+\delta \frac{(\psi * \phi) * f}{(\psi * \varphi) * f} \in \mathcal{K}_{\mu}(h)
$$

Thus, we have $f \in \mathcal{M}^{\delta}(\psi * \Phi, \xi, h)$ and, in consequence, we get (36). From (36) and Lemma 11 we have (37).

Theorem 12 Let $\psi, \xi \in \mathcal{R}_{p}(\alpha), 0 \leq \delta \leq 1$. Then

$$
\begin{aligned}
& \mathcal{C W}_{\mu}(\Phi, \mathbf{h}) \subset \mathcal{C} \mathcal{W}_{\mu}(\psi * \Phi, \mathbf{h}) \\
& \mathcal{C M}_{\mu}^{\delta}(\Phi, \xi, \mathbf{h}) \cap \mathcal{C} \mathcal{W}_{\mu}(\Phi, \mathbf{h}) \subset \mathcal{C} \mathcal{M}_{\mu}^{\delta}(\psi * \Phi, \xi, \mathbf{h})
\end{aligned}
$$

Proof Let $f \in \mathcal{C W}_{\boldsymbol{\mu}}(\Phi, \mathbf{h})$. Then there exist $g \in \mathcal{W}_{\mu_{2}}\left(\varphi, h_{2}\right)$ and $\omega_{1}, \omega_{2} \in \Omega$ such that

$$
\frac{\phi * f}{\varphi * g}=\mu_{1}\left(h_{1} \circ \omega_{1}\right)+\left(1-\mu_{1}\right)\left(h_{1} \circ \omega_{2}\right)
$$

Since $F=\varphi * g \in S_{p}^{*}(\alpha)$, applying the properties of convolution, we obtain

$$
\frac{(\psi * \phi) * f}{(\psi * \varphi) * g}=\mu_{1} \frac{\psi *\left[\left(h_{1} \circ \omega_{1}\right) F\right]}{\psi * F}+\left(1-\mu_{1}\right) \frac{\psi *\left[\left(h_{1} \circ \omega_{2}\right) F\right]}{\psi * F} .
$$

Analysis similar to that in the proof of Theorem 10 gives

$$
\frac{(\psi * \phi) * f}{(\psi * \varphi) * g} \in \mathcal{K}_{\mu_{1}}\left(h_{1}\right)
$$

Moreover, by Theorem 10 we have $g \in \mathcal{W}_{\mu_{2}}\left(\psi * \varphi, h_{2}\right)$ and, in consequence, $f \in \mathcal{C W}_{\mu}(\psi *$ $\Phi, \mathbf{h})$. This proves (38). To prove (39) we assume that $f \in \mathcal{C M}_{\mu}^{\delta}(\Phi, \xi, \mathbf{h}) \cap \mathcal{C} \mathcal{W}_{\mu}(\Phi, \mathbf{h})$. 
Then, applying (38) and Theorem 6 , we obtain $f \in \mathcal{C W}_{\mu}(\psi * \Phi, \mathbf{h})$ and $f \in \mathcal{C W}_{\mu}((\xi * \psi) *$ $\Phi, \mathbf{h})$ or, equivalently,

$$
q_{1}:=\frac{\psi * \phi * f}{\psi * \varphi * g} \in \mathcal{K}_{\mu_{1}}\left(h_{1}\right), \quad q_{2}:=\frac{\xi * \psi * \phi * f}{\xi * \psi * \varphi * g} \in \mathcal{K}_{\mu_{1}}\left(h_{1}\right) .
$$

Since the class $\mathcal{K}_{\mu_{1}}\left(h_{1}\right)$ is convex by Theorem 1 , we conclude that

$$
(1-\delta) \frac{\xi * \psi * \phi * f}{\xi * \psi * \varphi * g}+\delta \frac{\psi * \phi * f}{\psi * \varphi * g} \in \mathcal{K}_{\mu_{1}}\left(h_{1}\right)
$$

This gives (39) and completes the proof.

Combining Theorems 10-12 with Theorem 9, we obtain the following corollary.

Corollary 2 If either (24) or (25), then

$$
\begin{aligned}
& \mathcal{W}_{\mu}(\varphi, h) \subset \mathcal{W}_{\mu}\left(l_{p}(a, c) * \varphi, h\right), \\
& \mathcal{C W}_{\mu}(\Phi, \mathbf{h}) \subset \mathcal{C W}_{\mu}\left(l_{p}(a, c) * \Phi, \mathbf{h}\right), \\
& \mathcal{W}_{\mu}(\Phi, h) \cap \mathcal{W}_{\mu}(\varphi, h) \subset \mathcal{W}_{\mu}\left(l_{p}(a, c, z) * \Phi, h\right)
\end{aligned}
$$

Moreover, if $\xi \in \mathcal{R}_{p}(\alpha)$ and $0 \leq \delta \leq 1$, then

$$
\begin{aligned}
& \mathcal{M}_{1}^{\delta}(\varphi, h) \subset \mathcal{M}_{1}^{\delta}\left(l_{p}(a, c) * \varphi, h\right), \\
& \mathcal{M}_{\mu}^{\delta}(\Phi, \xi, h) \cap \mathcal{W}_{\mu}(\Phi, h) \subset \mathcal{M}_{\mu}^{\delta}\left(l_{p}(a, c) * \Phi, \xi, h\right), \\
& \mathcal{C M}_{\mu}^{\delta}(\Phi, \xi, \mathbf{h}) \cap \mathcal{C} \mathcal{W}_{\mu}(\Phi, \mathbf{h}) \subset \mathcal{C} \mathcal{M}_{\mu}^{\delta}\left(l_{p}(a, c) * \Phi, \xi, \mathbf{h}\right)
\end{aligned}
$$

Since $l_{p}(a, c) * l_{p}(c, a) * \phi=\phi$, by Theorem 2 we obtain the next result.

Corollary 3 If either (24) or (25), then

$$
\begin{aligned}
& \mathcal{W}_{\mu}\left(l_{p}(c, a) * \varphi, h\right) \subset \mathcal{W}_{\mu}(\varphi, h), \\
& \mathcal{C W}_{\mu}\left(l_{p}(c, a) * \Phi, \mathbf{h}\right) \subset \mathcal{C} \mathcal{W}_{\mu}(\Phi, \mathbf{h}), \\
& \mathcal{W}_{\mu}\left(l_{p}(c, a) * \Phi, h\right) \cap \mathcal{W}_{\mu}\left(l_{p}(c, a) * \varphi, h\right) \subset \mathcal{W}_{\mu}(\Phi, h) .
\end{aligned}
$$

Moreover, if $\xi \in \mathcal{R}_{p}(\alpha)$ and $0 \leq \delta \leq 1$, then

$$
\begin{aligned}
& \mathcal{M}_{1}^{\delta}\left(l_{p}(c, a) * \varphi, h\right) \subset \mathcal{M}_{1}^{\delta}(\varphi, h), \\
& \mathcal{M}_{\mu}^{\delta}\left(l_{p}(c, a) * \Phi, \xi, h\right) \cap \mathcal{W}_{\mu}\left(l_{p}(c, a) * \Phi, h\right) \subset \mathcal{M}_{\mu}^{\delta}(\Phi, \xi, h), \\
& \mathcal{C} \mathcal{M}_{\mu}^{\delta}\left(l_{p}(c, a) * \Phi, \xi, \mathbf{h}\right) \cap \mathcal{C W}_{\mu}\left(l_{p}(c, a) * \Phi, \mathbf{h}\right) \subset \mathcal{C M}_{\mu}^{\delta}(\Phi, \xi, \mathbf{h})
\end{aligned}
$$

Let us define the linear operators $J_{\lambda}: \mathcal{A}_{p} \longrightarrow \mathcal{A}_{p}, J_{\lambda}^{\times}: \mathcal{A}_{p} \times \mathcal{A}_{p} \longrightarrow \mathcal{A}_{p} \times \mathcal{A}_{p}$,

$$
\begin{aligned}
& J_{\lambda}(f)(z):=\lambda \frac{z f^{\prime}(z)}{p}+(1-\lambda) f(z), \\
& J_{\lambda}^{\times}(f, g):=\left(J_{\lambda}(f), J_{\lambda}(g)\right) \quad(z \in \mathcal{U}, \operatorname{Re} \lambda>0) .
\end{aligned}
$$


Since $J_{\lambda}(\varphi)=l_{p}\left(\frac{p}{\lambda}+1, \frac{p}{\lambda}\right) * \varphi$, putting $a=\frac{p}{\lambda}, c=\frac{p}{\lambda}+1$ in Corollary 3 , we have the following corollary.

Corollary 4 If $p-\operatorname{Re} p / \lambda \leq \alpha<p$, then

$$
\begin{aligned}
& \mathcal{W}_{\mu}\left(J_{\lambda}(\varphi), h\right) \subset \mathcal{W}_{\mu}(\varphi, h), \\
& \mathcal{C W}_{\mu}\left(J_{\lambda}^{\times}(\Phi), \mathbf{h}\right) \subset \mathcal{C} \mathcal{W}_{\mu}(\Phi, \mathbf{h}), \\
& \mathcal{W}_{\mu}\left(J_{\lambda}^{\times}(\Phi), h\right) \cap \mathcal{W}_{\mu}\left(J_{\lambda}(\varphi), h\right) \subset \mathcal{W}_{\mu}(\Phi, h)
\end{aligned}
$$

Moreover, if $\xi \in \mathcal{R}_{p}(\alpha)$ and $0 \leq \delta \leq 1$, then

$$
\begin{aligned}
& \mathcal{M}_{1}^{\delta}\left(J_{\lambda}(\varphi), h\right) \subset \mathcal{M}_{1}^{\delta}(\varphi, h), \\
& \mathcal{M}_{\mu}^{\delta}\left(J_{\lambda}^{\times}(\Phi), \xi, h\right) \cap \mathcal{W}_{\mu}\left(J_{\lambda}^{\times}(\Phi), h\right) \subset \mathcal{M}_{\mu}^{\delta}(\Phi, \xi, h), \\
& \mathcal{C} \mathcal{M}_{\mu}^{\delta}\left(J_{\lambda}^{\times}(\Phi), \xi, \mathbf{h}\right) \cap \mathcal{C} \mathcal{W}_{\mu}\left(J_{\lambda}^{\times}(\Phi), \mathbf{h}\right) \subset \mathcal{C} \mathcal{M}_{\mu}^{\delta}(\Phi, \xi, \mathbf{h}) .
\end{aligned}
$$

In particular, for $\lambda=1$, we get the following corollary.

Corollary 5 If $0 \leq \alpha<p$, then

$$
\begin{aligned}
& \mathcal{W}_{\mu}\left(p^{-1} z \varphi^{\prime}(z), h\right) \subset \mathcal{W}_{\mu}(\varphi, h), \\
& \mathcal{C} \mathcal{W}_{\mu}\left(p^{-1} z \Phi^{\prime}(z), \mathbf{h}\right) \subset \mathcal{C} \mathcal{W}_{\mu}(\Phi, \mathbf{h}), \\
& \mathcal{W}_{\mu}\left(p^{-1} z \Phi^{\prime}(z), h\right) \cap \mathcal{W}_{\mu}\left(p^{-1} z \varphi^{\prime}(z), h\right) \subset \mathcal{W}_{\mu}(\Phi, h) .
\end{aligned}
$$

Moreover, if $\xi \in \mathcal{R}_{p}(\alpha)$ and $0 \leq \delta \leq 1$, then

$$
\begin{aligned}
& \mathcal{M}_{1}^{\delta}\left(p^{-1} z \varphi^{\prime}(z), h\right) \subset \mathcal{M}_{1}^{\delta}(\varphi, h), \\
& \mathcal{M}_{\mu}^{\delta}\left(p^{-1} z \Phi^{\prime}(z), \xi, h\right) \cap \mathcal{W}_{\mu}\left(p^{-1} z \Phi^{\prime}(z), h\right) \subset \mathcal{M}_{\mu}^{\delta}(\Phi, \xi, h), \\
& \mathcal{C} \mathcal{M}_{\mu}^{\delta}\left(p^{-1} z \Phi^{\prime}(z), \xi, \mathbf{h}\right) \cap \mathcal{C} \mathcal{W}_{\mu}\left(p^{-1} z \Phi^{\prime}(z), \mathbf{h}\right) \subset \mathcal{C} \mathcal{M}_{\mu}^{\delta}(\Phi, \xi, \mathbf{h}) .
\end{aligned}
$$

\section{Applications to classes defined by linear operators}

The classes $\mathcal{M}_{\mu}^{\delta}(\Phi, \xi, h)$ and $\mathcal{C M}_{\mu}^{\delta}(\Phi, \xi, \mathbf{h})$ generalize well-known important classes, which were investigated in earlier works. Most of these classes were defined by using linear operators and special functions.

Let $A_{1}, \ldots, A_{q}$ and $B_{1}, \ldots, B_{s}(q, s \in \mathbb{N})$ be positive real numbers such that

$$
1+\sum_{k=1}^{s} B_{k}-\sum_{k=1}^{q} A_{k} \geq 0
$$

For complex parameters $a_{1}, \ldots, a_{q}$ and $b_{1}, \ldots, b_{s}(q, s \in \mathbb{N})$ such that $\frac{a_{k}}{A_{k}}, \frac{b_{k}}{B_{k}} \neq 0,-1,-2, \ldots$, we define the Fox-Wright generalization of the hypergeometric function by

$$
{ }_{q} \Psi_{s}\left[\begin{array}{c}
\left(a_{1}, A_{1}\right), \ldots,\left(a_{q}, A_{q}\right) ; \\
\left(b_{1}, B_{1}\right), \ldots,\left(b_{s}, B_{s}\right) ;
\end{array}\right]:=\sum_{n=0}^{\infty} \frac{\Gamma\left(a_{1}+A_{1} n\right) \cdots \Gamma\left(a_{q}+A_{q} n\right)}{\Gamma\left(b_{1}+B_{1} n\right) \cdots \Gamma\left(b_{s}+B_{s} n\right)} \frac{z^{n}}{n !} \quad(z \in \mathcal{U}) .
$$


If $A_{n}=1(n=1, \ldots, q)$ and $B_{n}=1(n=1, \ldots, s)$, then we obtain the generalized hypergeometric function

$$
{ }_{q} F_{s}\left(a_{1}, \ldots, a_{q} ; b_{1}, \ldots, b_{s} ; z\right):=\omega_{q} \Psi_{s}\left[\begin{array}{c}
\left(a_{1}, 1\right), \ldots,\left(a_{q}, 1\right) ; \\
\left(b_{1}, 1\right), \ldots,\left(b_{s}, 1\right) ;
\end{array}\right] \quad(z \in \mathcal{U}),
$$

where

$$
\omega=\frac{\Gamma\left(b_{1}\right) \cdots \Gamma\left(b_{s}\right)}{\Gamma\left(a_{1}\right) \cdots \Gamma\left(a_{q}\right)} .
$$

Moreover, in terms of Fox's $H$-function, we have

$$
{ }_{q} \Psi_{s}\left[\begin{array}{l}
\left(a_{1}, A_{1}\right), \ldots,\left(a_{q}, A_{q}\right) ; \\
\left(b_{1}, B_{1}\right), \ldots,\left(b_{s}, B_{s}\right) ;
\end{array}\right]=H_{q, s+1}^{1, q}\left[-z \mid \begin{array}{l}
\left(1-a_{1}, A_{1}\right), \ldots,\left(1-a_{q}, A_{q}\right) \\
(0,1)\left(1-b_{1}, B_{1}\right), \ldots,\left(1-b_{s}, B_{s}\right)
\end{array}\right] .
$$

Other interesting and useful special cases of the Fox-Wright function defined by (42) include (for example) the generalized Bessel function $J_{\nu}^{\mu}$ defined by

$$
J_{v}^{\mu}(z):=\sum_{n=0}^{\infty} \frac{(-z)^{n}}{n ! \Gamma(1+v+\mu n)} \quad(z \in \mathcal{U})
$$

which, for $\mu=1$, corresponds essentially to the classical Bessel function $J_{v}$, and the generalized Mittag-Leffler function $E_{\lambda, \mu}$ defined by

$$
E_{\lambda, \mu}(z):=\sum_{n=0}^{\infty} \frac{z^{n}}{\Gamma(v+\lambda n)} \quad(z \in \mathcal{U})
$$

For real numbers $\lambda, t(\lambda>-p)$, we define the function

$$
\zeta\left(a_{1}, b_{1}, t\right)(z):=\left(\omega z_{q}^{p} \Psi_{s}\left[\begin{array}{c}
\left(a_{1}, A_{1}\right), \ldots,\left(a_{q}, A_{q}\right) \\
\left(b_{1}, B_{1}\right), \ldots,\left(b_{s}, B_{s}\right)
\end{array} ; z\right]\right) * f_{\lambda, t}(z),
$$

where $\omega$ is defined by (43) and

$$
f_{\lambda, t}(z)=\sum_{n=p}^{\infty}\left(\frac{n+\lambda}{p+\lambda}\right)^{t} z^{n} \quad(z \in \mathcal{U})
$$

It is easy to verify that

$$
\begin{aligned}
& a \zeta(a+1, b, t)=A_{1} z \zeta^{\prime}(a ; b, t)+\left(a-p A_{1}\right) \zeta(a, b, t), \\
& b \zeta(a, b, t)=B_{1} z \zeta^{\prime}(a, b+1, t)+\left(b-p B_{1}\right) \zeta(a, b+1, t), \\
& (p+\lambda) \zeta(a, b, t+1)=z \zeta^{\prime}(a, b, t)+\lambda \zeta(a, b, t), \\
& \zeta(a, b, t)=l_{p}(a, c) * \zeta(c, b, t) \quad\left(A_{1}=1\right), \\
& \zeta(a, c, t)=l_{p}(b, c) * \zeta(a, b, t) \quad\left(B_{1}=1\right),
\end{aligned}
$$

where $l_{p}(a, c)$ is defined by (23). 
Concerning the function $\zeta(a, b, t)$, we consider the following classes of functions:

$$
\mathcal{V}(a, b, t):=\mathcal{W}_{\mu}(\zeta(a, b, t), h), \quad \mathcal{C} \mathcal{V}(a, b, t):=\mathcal{C W}_{\mu}(\zeta(a, b, t), \mathbf{h})
$$

By using the linear operator

$$
\Theta_{p}[a, b, t] f=\zeta(a, b, t) * f \quad\left(f \in \mathcal{A}_{p}\right)
$$

we can define the class $\mathcal{V}(a, b, t)$ alternatively in the following way:

$$
f \in \mathcal{V}(a, b, t) \Longleftrightarrow \frac{a}{A_{1}} \frac{\Theta_{p}[a+1, b, t] f(z)}{\Theta_{p}[a, b, t] f(z)}+p-\frac{a}{A_{1}} \in \mathcal{K}_{\mu}(h) .
$$

Corollary 6 If $p-\operatorname{Re} a / A_{1} \leq \alpha<p, m \in \mathbb{N}$, then

$$
\mathcal{V}(a+m, b, t) \subset \mathcal{V}(a, b, t), \quad \mathcal{C} \mathcal{V}(a+m, b, t) \subset \mathcal{C V}(a, b, t)
$$

Proof It is sufficient to prove the corollary for $m=1$. Let $J_{\lambda}$ and $\zeta(a, b, t)$ be defined by (41) and (44), respectively. Then by (45) we have $\zeta(a+1, b, t)=J_{\frac{p A_{1}}{a}}(\zeta(a, b, t))$. Hence, by using Corollary 4, we conclude that

$$
\begin{aligned}
& \mathcal{W}_{\mu}(\zeta(a+1, b, t), h) \subset \mathcal{W}_{\mu}(\zeta(a, b, t), h), \\
& \mathcal{C} \mathcal{W}_{\mu}(\zeta(a+1, b, t), \mathbf{h}) \subset \mathcal{C} \mathcal{W}_{\mu}(\zeta(a, b, t), \mathbf{h})
\end{aligned}
$$

This clearly forces the inclusion relations (48) for $m=1$.

Analogously to Corollary 6, we prove the following corollary.

Corollary 7 Let $m \in \mathbb{N}$. If $p-\operatorname{Re} b / B_{1} \leq \alpha<p$, then

$$
\begin{aligned}
& \mathcal{V}(a, b, t) \subset \mathcal{V}(a, b+m, t), \quad \mathcal{C} \mathcal{V}(a, b, t) \subset \mathcal{C} \mathcal{V}(a, b+m, t) . \\
& \text { If }-\operatorname{Re} \lambda \leq \alpha<p \text {, then } \\
& \mathcal{V}(a, b, t+m) \subset \mathcal{V}(a, b, t), \quad \mathcal{C V}(a, b, t+m) \subset \mathcal{C} \mathcal{V}(a, b, t) .
\end{aligned}
$$

It is natural to ask about the inclusion relations in Corollaries 6 and 7 when $m$ is positive real. Using Theorems 10 and 12, we shall give a partial answer to this question.

Corollary 8 If $l_{p}(a, c) \in \mathcal{R}_{p}(\alpha)$, then

$$
\begin{array}{lll}
\mathcal{V}(c, b, t) \subset \mathcal{V}(a, b, t), & \mathcal{C V}(c, b, t) \subset \mathcal{C V}(a, b, t) & \left(A_{1}=1\right), \\
\mathcal{V}(b, a, t) \subset \mathcal{V}(b, c, t), & \mathcal{C V}(b, a, t) \subset \mathcal{C V}(b, c, t) & \left(B_{1}=1\right)
\end{array}
$$

Proof Let us put $\psi=l_{p}(a, c), \varphi=\zeta(c, b, t)$. Then, by Theorem 10, Theorem 12 and relationship (46), we obtain

$$
\mathcal{W}_{\mu}(\zeta(c, b, t), h) \subset \mathcal{W}_{\mu}(\zeta(a, b, t), h), \quad \mathcal{C W}_{\mu}(\zeta(c, b, t), \mathbf{h}) \subset \mathcal{C} \mathcal{W}_{\mu}(\zeta(a, b, t), \mathbf{h})
$$

Thus, we get the inclusions (49). Analogously, we prove the inclusions (50). 
Combining Corollary 8 with Theorem 9, we obtain the following result.

Corollary 9 If either (24) or (25), then the inclusion relations (49) and (50) hold true.

The linear operator $\Theta_{p}[a, b, t]$ defined by (47) includes (as its special cases) other linear operators of geometric function theory which were considered in earlier works. In particular, the operator $\Theta_{p}[a, b, 0]$ was introduced by Dziok and Raina [11]. It contains, as its further special cases, such other linear operators as the Dziok-Srivastava operator, the Carlson-Shaffer operator, the Ruscheweyh derivative operator, the generalized BernardiLibera-Livingston operator (for the precise relationships, see Dziok and Srivastava ([13], pp.3-4)). Moreover, the linear operator $\Theta_{p}[a, b, t]$ includes also the Sălăgean operator, the Noor operator, the Choi-Saigo-Srivastava operator (for the precise relationships, see Cho et al. [4]). By using these linear operators, we can consider some subclasses of the classes $\mathcal{V}(a, b, t), \mathcal{C V}(a, b, t)$, see for example $[2-5,7-9,12,15,30,32,36-38]$. Also, the obtained results generalize several results obtained in these classes of functions.

\section{Competing interests}

The author declares that they have no competing interests .

Received: 1 May 2013 Accepted: 10 July 2013 Published: 26 July 2013

\section{References}

1. Mocanu, PT: Une propriété de convexité g énéralisée dans la théorie de la représentation conforme. Mathematica (Cluj, 1929) 11(34), 127-133 (1969) (in French)

2. Aouf, MK: Some inclusion relationships associated with Dziok-Srivastava operator. Appl. Math. Comput. 216, 431-437 (2010)

3. Aouf, MK, Seoudy, TM: Some properties of certain subclasses of $p$-valent Bazilevic functions associated with the generalized operator. Appl. Math. Lett. 24(11), 1953-1958 (2011)

4. Cho, NE, Kwon, OS, Srivastava, HM: Inclusion relationships and argument properties for certain subclasses of multivalent functions associated with a family of linear operators. J. Math. Anal. Appl. 292, 470-483 (2004)

5. Choi, JH, Saigo, M, Srivastava, HM: Some inclusion properties of a certain family of integral operators. J. Math. Anal. Appl. 276, 432-445 (2002)

6. Coonce, HB, Ziegler, MR: Functions with bounded Mocanu variation. Rev. Roum. Math. Pures Appl. 19, 1093-1104 (1974)

7. Dziok, J: Applications of multivalent prestarlike functions. Appl. Math. Comput. (to appear)

8. Dziok, J: Applications of the Jack lemma. Acta Math. Hung. 105, 93-102 (2004)

9. Dziok, J: Inclusion relationships between classes of functions defined by subordination. Ann. Pol. Math. 100, 193-202 (2011)

10. Dziok, J: Characterizations of analytic functions associated with functions of bounded variation. Ann. Pol. Math. 109, 199-207 (2013)

11. Dziok, J, Raina, RK: Families of analytic functions associated with the wright generalized hypergeometric function. Demonstr. Math. 37, 533-542 (2004)

12. Dziok, J, Sokół, J: Some inclusion properties of certain class of analytic functions. Taiwan. J. Math. 13, 2001-2009 (2009)

13. Dziok, J, Srivastava, HM: Classes of analytic functions associated with the generalized hypergeometric function. Appl. Math. Comput. 103, 1-13 (1999)

14. Eenigenburg, PJ, Miller, SS, Mocanu, PT, Reade, OM: Second order differential inequalities in the complex plane. J. Math. Anal. Appl. 65, 289-305 (1978)

15. Liu, J-L, Noor, Kl: On subordinations for certain analytic functions associated with Noor integral operator. Appl. Math. Comput. 187, 1453-1460 (2007)

16. Liu, M-S, Zhu, Y-C, Srivastava, HM: Properties and characteristics of certain subclasses of starlike functions of order $\beta$. Math. Comput. Model. 48, 402-419 (2008)

17. Lyzzaik, A: Multivalent functions of bounded boundary rotation and weakly close-to-convex functions. Proc. Lond. Math. Soc. 51(3), 478-500 (1985)

18. Hallenbeck, DJ, MacGregor, TH: Linear Problems and Convexity Techniques in Geometric Function Theory, Pitman Advanced Publishing Program. Pitman, Boston (1984)

19. Jack, IS: Functions starlike and convex of order $\alpha$. J. Lond. Math. Soc. 3, 469-474 (1971)

20. Miller, SS, Mocanu, PT: Differential Subordinations: Theory and Applications. Series on Monographs and Textbooks in Pure and Applied Mathematics, vol. 225. Dekker, New York (2000)

21. Moulis, EJ: Generalizations of the Robertson functions. Pac. J. Math. 81, 167-174 (1979)

22. Noor, Kl: On some subclasses of functions with bounded radius and bounded boundary rotation. Panam. Math. J. 6(1), 75-81 (1996)

23. Noor, Kl: On uniformly Bazilevic and related functions. Abstr. Appl. Anal. 2012, Article ID 345261 (2012) 
24. Noor, Kl, Hussain, S: On certain analytic functions associated with Ruscheweyh derivatives and bounded Mocanu variation. J. Math. Anal. Appl. 340(2), 1145-1152 (2008)

25. Noor, Kl, Malik, SN: On generalized bounded Mocanu variation associated with conic domain. Math. Comput. Model. 55(3-4), 844-852 (2012)

26. Noor, Kl, Muhammad, A: On analytic functions with generalized bounded Mocanu variation. Appl. Math. Comput. 196(2), 802-811 (2008)

27. Noor, KI, Ul-Haq, W: On some implication type results involving generalized bounded Mocanu variations. Comput. Math. Appl. 63(10), 1456-1461 (2012)

28. Özkan, Ö, Altıntaş, O: Applications of differential subordination. Appl. Math. Lett. 19, 728-734 (2006)

29. Paatero, V: Über die konforme Abbildung von Gebieten deren Ränder von beschränkter Drehung sind. Ann. Acad. Sci. Fenn., A 33, 1-79 (1931)

30. Patel, J, Mishra, AK, Srivastava, HM: Classes of multivalent analytic functions involving the Dziok-Srivastava operator. Comput. Math. Appl. 54, 599-616 (2007)

31. Padmanabhan, K, Parvatham, R: Properties of a class of functions with bounded boundary rotation. Ann. Pol. Math. 31, 311-323 (1975)

32. Piejko, K, Sokół, J: On the Dziok-Srivastava operator under multivalent analytic functions. Appl. Math. Comput. 177, 839-843 (2006)

33. Pinchuk, B: Functions with bounded boundary rotation. Isr. J. Math. 10, 7-16 (1971)

34. Ruscheweyh, S: Convolutions in Geometric Function Theory. Sem. Math. Sup., vol. 83. Presses University Montreal, Montreal (1982)

35. Ruscheweyh, S: Linear operators between classes of prestarlike functions. Comment. Math. Helv. 52, 497-509 (1977)

36. Sokół, J, Trojnar-Spelina, L: Convolution properties for certain classes of multivalent functions. J. Math. Anal. Appl. 337, 1190-1197 (2008)

37. Srivastava, HM, Lashin, AY: Subordination properties of certain classes of multivalently analytic functions. Math Comput. Model. 52, 596-602 (2010)

38. Wang, Z-G, Zhang, G-W, Wen, F-H: Properties and characteristics of the Srivastava-Khairnar-More integral operator. Appl. Math. Comput. 218, 7747-7758 (2012)

doi:10.1186/1029-242X-2013-349

Cite this article as: Dziok: Classes of functions associated with bounded Mocanu variation. Journal of Inequalities and Applications 2013 2013:349. 